\title{
Exogenous Recombinant Adiponectin Improves Survival in Experimental Abdominal Sepsis
}

\author{
Bülent Salman ${ }^{1}$, Tonguç Utku Y1lmaz², Tugan Tezcaner ${ }^{3}$, Ebru Ofluoğlu Demir ${ }^{4}$, Özge Tuğçe Paşaoğlu \\ ${ }^{1}$ Department of General Surgery, Gazi University Faculty of Medicine, Ankara, Turkey \\ ${ }^{2}$ Department of General Surgery, Kocaeli Universtiy Faculty of Medicine, Kocaeli, Turkey \\ ${ }^{3}$ Department of General Surgery, Başkent University Faculty of Medicine, Ankara, Turkey \\ ${ }^{4}$ Department of Medical Serivices and Techniques, Bülent Ecevit University Vocational School of Health Services, Zonguldak, Turkey \\ ${ }^{5}$ Department of Biochemistry, Gazi University Faculty of Health Sciences, Ankara, Turkey
}

Background: Adiponectin, which has anti-inflammatory features, is an important substance in several metabolic mechanisms.

Aims: The aim of this study is to evauate the effects of exogenous intraperitoneal administration of adiponectin on the survival, intrabdominal adhesion and inflammatory cytokine levels in an experimental sepsis model.

Study Design: Animal experimentation.

Methods: Ninety rats were divided into a control group, adiponectin group and sham group. A cecal puncture abdominal sepsis model was performed in the adiponectin and control groups. Every three hours, exogenous adiponectin was administrated to the adiponectin group. At the $3^{\text {rd }}$ and $24^{\text {th }}$ hours, 10 rats were sacrified in each group in order to measure plasma tumor necrosis factor- $\alpha$ (TNF- $\alpha$ ), interleukin (IL) 10, soluble intracellular adhesion molecule (ICAM)-1, IL-6 and macrophage inhibitory factor levels, and the activity of nuclear factor (NF)-kB. The remaining rats were followed for survival.

Results: The plasma levels of TNF- $\alpha$, soluable ICAM-1, IL-6, and macrophage inhibitory factor were significantly higher in the control group than in the adiponectin and sham group $(\mathrm{p}<0.05)$. The increase in inflammatory cytokines with time was more prominent in the control group. The activity of NF-kB in the control group was higher than in the adiponectin group $(\mathrm{p}<0.05)$. The survival rate of the adiponectin group was higher than in the control group.

Conclusion: Administration of exogenous adiponectin to the peritoneum in abdominal sepsis increased survival and decreased intrabdominal adhesions by decreasing the inflammatory response.

(Balkan Med J 2014;31:244-8).

Key Words: Adhesion, adiponectin, cytokine, sepsis, survival
Bacterial sepsis is the major cause of mortality and morbidity among patients (1). The conventional therapy for sepsis includes maintenance of tissue perfusion and treatment of infections. Several pathophysiological pathways that have been determined to be involved in sepsis involve an imbalance between inflammatory and immune responses; these pathways were targeted. Cytokines, especially tumor necrosis factor- $\alpha$ (TNF- $\alpha$ ) and interleukin (IL)-6, together with oxygen free radicals, are believed to be the responsible factors in tissue injury that precedes multiple organ dysfunction with activation of the endothelial system and vascular dysfunction (2). One of the molecules that increases macrophage binding and activation is intracellular adhesion molecule (ICAM-1), which is closely linked to the inflammatory response. Beside the cytokines and adhesion molecules that play a role in the inflammatory response, macrophage migration inhibitory factor (MIF), which plays a role in native immunity, plays a crucial role in sepsis mechanisms as a proinflammatory cytokine (3). Proinflammatory cytokine expression is increased by MIF, which is related to mortality in sepsis. From this point of view, the removal or neutralisation of inflammatory cytokines and ICAM molecules and depression of MIF is one approach to sepsis treatment.

Several inflammatory cytokines, especially TNF- $\alpha$, act by using a nuclear factor (NF)-kB pathway (4). Activation of NF$\mathrm{kB}$ leads to the secretion of several inflammatory cytokines, chemokines, enzymes, and adhesion molecules. Inhibition of NF-kB activity might therefore decelerate inflammation.

Adiponectin (APN), an adipocyte-derived protein, shows its anti-inflammatory properties by antagonizing the effects of TNF- $\alpha$ (5). Also, it has been shown that APN neutralizes endotoxin activities in vitro and slows the progression of polymicrobial sepsis in rats (6). In experimental and clinical studies, a negative corelation between plasma APN levels and 
TABLE 1. The mean levels of TNF- $\alpha$, IL-6, IL-10, ICAM-1 and MIF and the activity of NF-kB and overall means are given at the $3^{\text {rd }}$ and $24^{\text {th }}$ hours

\begin{tabular}{|c|c|c|c|c|c|c|}
\hline & $\mathrm{TNF}-\alpha(\mathrm{pg} / \mathrm{mL})$ & IL-6 (pg/mL) & IL-10 (pg/mL) & ICAM-1 (ng/mL) & $\operatorname{MIF}(\mathrm{pg} / \mathrm{mL})$ & NF-kB (\%) \\
\hline Sham $(n=30)$ & $4.5 \pm 1.2$ & $92 \pm 11$ & $174 \pm 8.4$ & $137.2 \pm 6.3$ & $156.5 \pm 34.2$ & 4 \\
\hline \multicolumn{7}{|l|}{ Control $(n=30)$} \\
\hline $3^{\text {rd }}$ hour $(n=10)$ & $26.4 \pm 4.5$ & $254 \pm 9$ & $94.8 \pm 9$ & $245.5 \pm 7.4$ & $941.6 \pm 42.2$ & 43 \\
\hline $24^{\text {th }}$ hour $(\mathrm{n}=10)$ & $37.5 \pm 6.5$ & $323.5 \pm 15$ & $59.5 \pm 8.1$ & $313.4 \pm 5.4$ & $837.2 \pm 32.2$ & 35 \\
\hline Overall $(n=20)$ & $31.5 \pm 6.1$ & $298 \pm 24$ & $89.2 \pm 9.8$ & $279.4 \pm 6.1$ & $889.4 \pm 36.4$ & 40 \\
\hline \multicolumn{7}{|l|}{$\operatorname{APN}(n=30)$} \\
\hline $3^{\text {rd }}$ hour $(n=10)$ & $12.3 \pm 3.5$ & $112.4 \pm 5.5$ & $165.4 \pm 4.5$ & $163.4 \pm 3.2$ & $803.7 \pm 25.2$ & 12 \\
\hline $24^{\text {th }}$ hour $(n=10)$ & $18.5 \pm 4.2$ & $174.5 \pm 6.2$ & $133.5 \pm 4.1$ & $202.4 \pm 4.3$ & $682.1 \pm 32.1$ & 8 \\
\hline Overall $(n=20)$ & $15.5 \pm 3.2$ & $154.2 \pm 18$ & $158 \pm 24.4$ & $182.9 \pm 3.6$ & $742.9 \pm 28.1$ & 10 \\
\hline
\end{tabular}

TNF- $\alpha$ : Tumor necrosis factor- $\alpha$; IL: interleukin; ICAM: Intracellular adhesion molecule; MIF: macrophage migration inhibitory factor; NF-кB: nuclear factor kappa B; pg: picogram; ng: nanogram; mL: mililitre

plasma TNF- $\alpha$ levels in sepsis has been shown $(7,8)$. However, details of the effects and intracellular mechanisms of action of APN remain obscure. For this reason, we hypothesize that administration of APN in experimental intrabdominal sepsis could influence the anti-inflammatory effect. The aim of this study was to evaluate the effects of APN on inflammatory cytokines, ICAM-1, MIF, and NF-kB and survival in a cecal ligation and puncture model.

\section{MATERIAL AND METHODS}

\section{Animals}

All procedures were performed according to the guidelines of the Care and Use of Experimental Animals and were approved by the Local Ethics Commitee. A total of 90 rats were kept at $21^{\circ} \mathrm{C}$ with a 12 -h light and dark cycle for 1 week before the experiment. All rats were fasted overnight for 12 hours with access to water ad libitum prior to the induction of sepsis.

\section{Experimental protocol}

The rats were randomly divided into 3 groups: Sham group $(n=30)$, Control group $(n=30)$, and APN group $(n=30)$. Anaesthetized $(50 \mathrm{mg} / \mathrm{kg}$ ketamine i.m; ketalar (10\% Ketamidor, Richter Pharma Co KG, Wels, Austria) rats underwent midline laparotomy. We isolated the cecum and than ligated $1 \mathrm{~cm}$ below the ileocecal valve using $3 / 0$ silk suture. The cecum was punctured two times with a 22 -gauge needle. Intraperitoneal recombinant adiponectin $(2 \mu \mathrm{g} / \mathrm{kg}$ ) (Biovision, CA, USA) was given to the rats in the APN group. The rats in the sham group underwent laparotomy only. Before closure of the abdomen, $3 \mathrm{~mL} / 100 \mathrm{~g}$ of saline was injected into the intraperitoneum. Every 3 hours, rats in the control group received $1 \mathrm{~mL}$ of intraperitoneal saline, while rats in the APN group received intraperitoneal APN $(2 \mu \mathrm{g} / \mathrm{kg})$. On the $6^{\text {th }}$ hour after the cecum ligation and puncture (CLP), 10 rats in the control group and 10 rats in the APN group were sacrified under ketamine anaesthe- sia. Ten rats in sham group were sacrified at the $3^{\text {rd }}$ hour after the laparotomy. Ten more rats in the control and APN groups were sacrified at the $24^{\text {th }}$ hour after CLP. Cecum necrosis was seen in all rats in the laparotomy group at the $3^{\text {rd }}$ and $24^{\text {th }}$ hours. The degree of intraperitoneal adhesion was classified as either: 0 : no adhesion, 1: adhesion in the lower abdomen, 2 : adhesion in the upper abdomen. The adhesions were evaluated during the laparotomy at the $24^{\text {th }}$ hour. Blood samples were taken from all rats through the inferior vena cava. Centrifugation at $3000 \mathrm{rpm}$ for 10 minutes at $4^{\circ} \mathrm{C}$ was performed in order to seperate the plasma. The plasma was stored at $-80^{\circ} \mathrm{C}$. We measured plasma IL-6, TNF- $\alpha$, IL-10, soluble ICAM-1, and macrophage inhibitory factor using a specific enzyme-linked immunosorbent assay (ELISA) kit (Biosource International, Camarillo, CA, USA). The NF- $\mathrm{kB}$ activity was measured using the method described by Arnalich F et al. (9).

The remaining 30 rat were kept under standard conditions, allowed free access to food and water ad libitum and followed until death or for a period of 6 days. The time from the end of CLP to death was accepted as the survival time of the rats.

\section{Data analysis}

Data from the study are presented as mean $\pm \mathrm{SD}$ and were analysed by Statistical Package for the Social Sciences (SPSS) version 14.00 (SPSS, Chicago, IL, USA). A one-way analysis of variance (ANOVA) with postHoc Scheffy was used for group comparisons. A bivariant Pearson correlation test was used for group correlations. The level of statistical significance was set at $p<0.05$. The survival rate was measured by the Kaplan-Meier method and compared using the log rank test.

\section{RESULTS}

The mean levels of data at the $3^{\text {rd }}$ and $24^{\text {th }}$ hours for each group and the means of all data in the groups were evaluated. The levels are given in Table 1. 


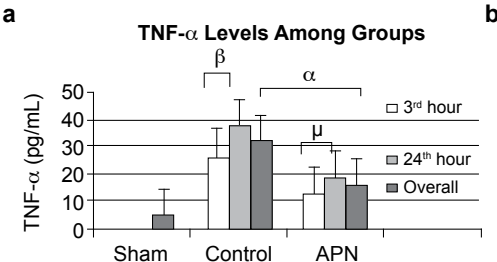

d

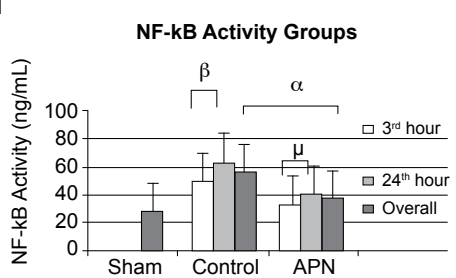

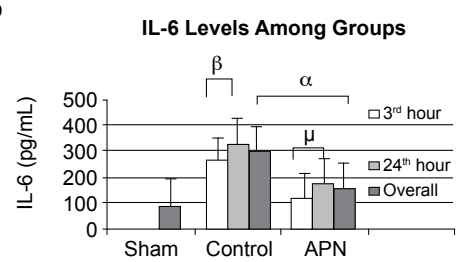

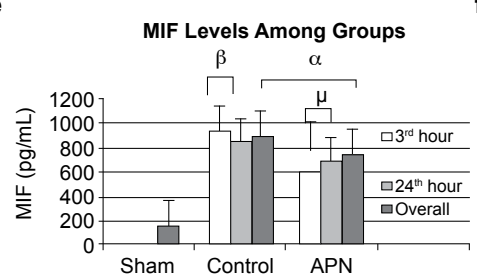

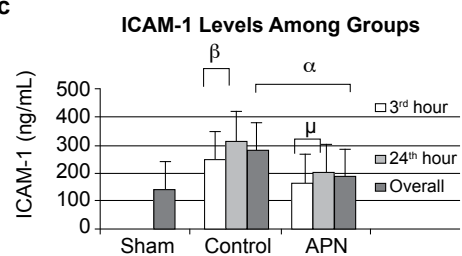

f

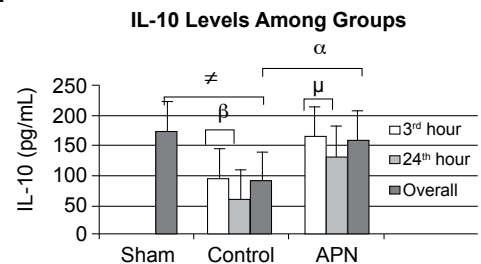

FIG. 1. a-f. The levels of TNF- $\alpha, I L-6, I L-10, I C A M-1$ and MIF at the $3^{\text {rd }}$ and $24^{\text {th }}$ hours. The overall means are also given. $P$ values of the comparisons were pointed by the $\alpha, b$, and $\mu$. NF-кB activity in the different groups, given as percentages. TNF- $\alpha$ levels in the groups at the $3^{\text {rd }}$ and $24^{\text {th }}$ hours. $\beta: p=0.01, \alpha: p=0.001, \mu: p=0.03$ (a). IL-6 levels in the groups at the $3^{\text {rd }}$ and $24^{\text {th }}$ hours. $\beta: p=0.01, \alpha: p=0.001, \mu: p=0.03$ (b). ICAM-1 levels in the groups at the $3^{\text {rd }}$ and $24^{\text {th }}$ hours. $\beta: p=0.02, \alpha: p=0.001, \mu: p=0.04$ (c). NF-kB activity in the groups at the $3^{\text {rd }}$ and $24^{\text {th }}$ hours. $\beta: p=0.67, \alpha: p=0.001, \mu: p=0.56$ (d). MIF levels in the groups at the $3^{\text {rd }}$ and $24^{\text {th }}$ hours. $\beta: p=0.122, \alpha: p=0.01, \mu: p=0.09$ (e). IL-10 levels in the groups at the $3^{\text {rd }}$ and $24^{\text {th }}$ hours. $\beta: p=0.01, \alpha: p=0.005, \mu: p=0.03, \#: p=0.001$ (f)

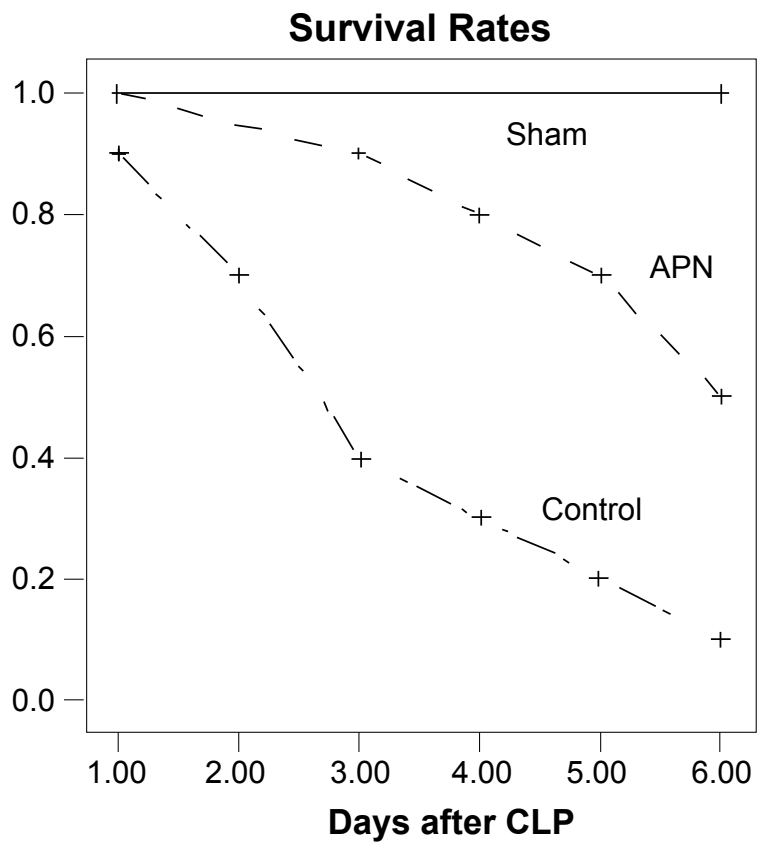

FIG. 2. Differences in survival curves produced by the administration of APN. The survival rates after CLP in the sham, APN and control groups. Differences between the survival rate $s$ in each group were analyzed by the log-rank test. Administration of APN significantly increased survival time when the APN group was compared with the control group $(p<0.001)$

There were significant differences between groups regarding the overall mean levels of TNF- $\alpha$, IL-6, ICAM1 and MIF $(\mathrm{p}<0.05)$ (Figure 1). The overall mean levels were significantly increased in the control group when compared with the APN and the sham group $(\mathrm{p}<0.05)$. On the other hand, the levels in the APN group were significantly higher than in the sham group but significantly lower than in the control group $(\mathrm{p}<0.05)$. When the mean levels of TNF- $\alpha$, IL- 6 , and ICAM- 1 at the $3^{\text {rd }}$ and $24^{\text {th }}$ hours were compared, the levels significantly increased with time $(\mathrm{p}<0.05)$. However, the increases in TNF- $\alpha$ $(\mathrm{r}=0.022)$, IL-6 $(\mathrm{r}=0.034)$, and ICAM-1 $(\mathrm{r}=0.028)$ levels in the control group were not correlated with those in the APN group. The increases in the APN group were smaller than in the control group.

The levels of IL-10 in the control group were significantly lower than in the sham and APN groups $(\mathrm{p}<0.05)$. On the other hand, the difference between the level of IL-10 in the APN and control groups was not significant. The levels of IL-10 and MIF decreased at the $24^{\text {th }}$ hour. While the difference between the levels at the $3^{\text {rd }}$ and $24^{\text {th }}$ hours was significant for IL-10, the difference was not significant for MIF. There were also significant differences in the survival rates between groups. In our study, NF-kB activity was significantly increased in the control group compared with the sham group. However, NF-kB activity was decreased in the APN group compared with the control group. On the other hand, NF-kB activities between the $3^{\text {rd }}$ and $24^{\text {th }}$ hours did not show any significant changes.

The mean intraabdominal adhesion scores of the sham, control and APN groups were $0.4 \pm 0.6,1.7 \pm 0.4$, and $1.2 \pm 0.4$, respectively. The mean adhesion score in the APN group was significantly higher than the mean adhesion score of the con- 
trol group. At the end of a six-day follow-up, survival was found to be improved in the APN group compared with the control group ( $<<0.001$; Figure 2$)$.

\section{DISCUSSION}

In our study, we found that the administration of intraperitoneal APN decreased inflammatory cytokines, increased an antiinflammatory cytokine, and decreased intrabdominal adhesions in an experimental intrabdominal sepsis model, and we demonstrated that APN supplementation might be a promising therapy for sepsis. As the CLP septic model mimics clinically observed sepsis with the same inflammatory and pathological sequences including Lipopolysaccharide (LPS) mediated reactions, we used the CLP model in order to evaluate the effects of APN.

Adiponectin has been studied for its role in glucose metabolism and it has been noted that APN levels are decreased in obese patients, patients suffering from coronary artery disease, and diabetic patients (10). APN also has important relationships in inflammatory reactions. There have been several studies showing that APN is an anti-inflammatory substance $(5,8)$. In in vitro studies, it was shown that administration of APN to cultured macrophages supressed the macrophages phagocytosis and LPS-induced TNF- $\alpha$ production. Also, it was demonstrated that the human recombinant APN directly bound LPS and suppressed limulus amoebocyte lysate activity in vitro $(6,11)$. Similarly, our experimental study showed that the APN treatment decreased TNF- $\alpha$, IL-6, and MIF levels while APN increased the levels of IL-10.

It was further shown that APN deficiency led to increased levels of plasma TNF- $\alpha$ and IL-6 in an experimental sepsis model obtained by CLP. Decreased APN led to increased susceptibility to polymicrobial sepsis with higher mortality and increased TNF- $\alpha$ and IL-6 levels (8). It was seen that while the levels of APN decreased, the plasma TNF- $\alpha$ levels increased, along with the lessening of sepsis. In our study, as sepsis progressed, TNF- $\alpha$ and IL- 6 levels increased in the control group, but the increase was significantly lower and slower in the APN group. These results showed that APN interacts by neutralizing LPS. Our study showed that APN decreased the inflammatory response and also decelerated the progression of sepsis. Low levels of APN seen in sepsis lead to excessive amounts of IL-6 and TNF- $\alpha$, which suppress APN production in the adipose tissue (12). The endotoxin, which had been released after the onset of intra-abdominal sepsis, was neutralized by several proteins such as albumin and LPS-binding proteins, but the remainder of the proteins activated macrophages and induced inflammatory mediators. A lack of APN has also been shown to increase the amount of endotoxin in CLP models (8). Furthermore, a key anti-inflammatory cytokine (IL-10) was decreased in sepsis in our study, but the APN inhibited the decrease in
IL-10. This result further supports the anti-inflammatory effects of APN. The neutralization of free endotoxins by APN might diminish inflammatory cytokine levels, as a deficiency in APN led to an increased inflammatory response and mortality. Local administration of APN in abdominal sepsis produced beneficial effects in our study. However, intravenous administration of APN should be the subject of future studies. Decreased adhesion in APN is a sign of a decreased inflammatory response in the abdomen. This is also another subject for future studies on the local antiinflamatory effects of APN.

In previous studies, several factors were shown to decrease the APN levels in sepsis. Endothelial injury, high levels of catecholamines, glucocorticoids, and IL-6 levels are seen in polymicrobial sepsis and all suppressed production of APN (13). For this reason, it is reasonable to administer APN. It was observed that Rosiglitazone augmented plasma APN levels when administered before the sepsis. In this way, a significantly decreased mortality rate was achieved in an experimental model (14). In our study, administration of APN, which is different from the previous studies, improved survival. Administration of APN, just with the onset of sepsis with repetitive doses during sepsis, resulted in a beneficial effect not only on local adhesions but also on sepsis. This finding shows us that the benefits of APN are not only expressed before the induction of sepsis but also during the progression of sepsis. APN supplementation with repetitive doses might be an encouraging treatment for patients with low levels of APN or for patients who are in the early phases of sepsis.

Besides its effects on LPS, APN affects endothelial function. Impaired endothelial function and leukocyte-endothelial interactions have been demonstrated in APN-deficient mice (15). Furthermore, APN deficiency resulted in increased recruitment of neutophils in the peritoneal cavity and more endothelial cell adhesion molecules (ICMA-1, VCAM-1) and exaggrated cytokine production in a peritonitis model (14). The circulating neutrophils were attached to the endothelial cells and became activated with the help of adhesion molecules, including ICAM-1. Adhesion molecules (ICAM-1, VCAM-1) were found to be increased in sepsis (16). Morphological changes with the activation of inflammatory cells lead to further inflammatory reactions. The effects of APN on monocytes decreased phagocytic activity, decreased adherence, and decreased lipid content. All of these results produced a decreased inflammatory response. By administering APN, we might favor its anti-inflammatory effects on monocytes as well. In our study, ICAM-1 levels increased significantly with sepsis progression and APN decelerated the increase. Our results support the beneficial effects of APN over endothelial function in sepsis. The result of the influence of APN on endothelium in our study was decreased adhesion. This shows us that APN also has a local antiinflammatory effect on endothelium. 
Migration inhibitory factor activates T-cells and macrophages in response to sepsis. Although it is a response to sepsis, increased levels of MIF are closely related to the severity of sepsis. In early phases, MIF levels are prognostic factors for the severity of sepsis (17). Improved survival was achieved by neutralizing MIF in an experimental sepsis model. In our study, APN decreased MIF levels when compared with the control group. Both ICAM-1 and MIF are related to NF-kB activity. This suggested that the effect of APN is related to NF-kB activity. In previous studies, it was shown that primary APN receptors led to the activation of several pathways, including AMP kinase and p38 mitogen-activated kinase (18). On the other hand, non-receptor-mediated events were also noted in association with high circulating levels of APN (14). Although the effects of APN on intracellular pathways are not yet clear, this field is another subject of research. The decreased NF-kB activity seen in our study is probably due to decreased stimulatory effects. In addition to these results, administration of APN increased survival rates significantly.

Although further studies are required to explain the intraractions between APN and sepsis-related changes, this study showd that APN has both local and systemic anti-inflammatory effects in an experimental sepsis model. Administration of APN in experimental abdominal sepsis decreased inflammatory cytokines, decreased intrabdominal adhesions and improved survival with its anti-inflammatory effects. This finding might be promising for the treatment of intrabdominal sepsis.

Ethics Committee Approval: Ethics committee approval was received for this study from local ethics committee.

\section{Informed Consent: N/A.}

Peer-review: Externally peer-reviewed.

Author contributions: Concept - B.S., T.U.Y., T.T.; Design - B.S., E.O.D., Ö.T.P.; Supervision - B.S.; Resource - B.S., T.T.; Materials - E.O.D., Ö.T.P.; Data Collection\&/or Processing - T.U.Y., T.T.; Analysis\&/or Interpretation B.S., T.U.Y., E.O.D.; Literature Search - T.U.Y., T.T.; Writing - B.S., T.U.Y.; Critical Reviews - B.S.

Acknowledgements: We want to thank to Mustafa KEREM who helped us for the support in experimental design.

Conflict of Interest: No conflict of interest was declared by the authors.

Financial Disclosure: This study was supported by Gazi University Scientific Research Project Unit.

\section{REFERENCES}

1. Annane D, Bellissant E, Cavaillon JM. Septic shock. Lancet 2005;365:63-78. [CrossRef]
2. Bozza FA, Salluh JI, Japiassu AM, Soares M, Assis EF, Gomes RN, et al. Cytokine profiles as markers of disease severity in sepsis: A multiple analysis. Crit Care 2007;11:R49. [CrossRef]

3. Baugh JA, Bucala R. Macrophage migration inhibitory factor. Crit Care Med 2002;30:27-35. [CrossRef]

4. Bedirli A, Salman B, Pasaoglu H, Ofluoglu E, Sakrak O. Effects of nuclear faktor-kB inhibitors on colon anastomic healing rats. J Surg Res 2011;171:355-60. [CrossRef]

5. Ouchi N, Walsh K. Adiponectin as an anti-inflammatory factor. Clin Chim Acta 2007;380:24-30. [CrossRef]

6. Tsuchihashi H, Yamamoto H, Maeda K, Ugi S, Shimizu T, Endo Y, et al. Circulating concentrations of adiponectin, an endogenous lipopolysaccharide neutralizing protein, decrease in rats with polymicrobial sepsis. J Sur Res 2006;134:348-53. [CrossRef]

7. Uji Y, Yamamoto H, Tsuchihashi H, Maeda K, Funahashi T, Shimomura $\mathrm{I}$, et al. Adiponectin deficiency is associated with severe polymicrobial sepsis, high inflammatory cytokine levels, and high mortality. Surgery 2009; 145:550-7. [CrossRef]

8. Uji Y, Yamamoto H, Maeda K, Tsuchihashi H, Akabori H, Shimizu T, et al. Adiponectin deficiency promotes the production of inflammatory mediators while severely exacerbating hepatic injury in mice with polymicrobial sepsis. J Surg Res 2010;161:301-11. [CrossRef]

9. Arnalich F, Garcia-Palomero E, Lopez J, Jimenez M, Maderoo R, Renart $\mathrm{J}$, et al. Predictive value of nuclear factor $\mathrm{kB}$ activity and plasma cytokine levels in patients with sepsis. Infect Immun 2000;68:1942-5. [CrossRef]

10. Söylemez N, Demirbağ R, Sezen Y, Yıldız A, Akpınar O. The levels of the leptin and adiponectin according to body mass index and their relationship with oxidative parameters. Anadolu Kardiyol Derg 2010;10:391-6. [CrossRef]

11. Yokota T, Oritani K, Takahashi I, Ishikawa J, Matsuyama A, Ouchi N, et al. Adiponectin, a new member of the family of solubale defense collagens, negatively regulates the growth of myelomonocytic progenitors and the functions of macrophages. Blood 2000;96:1723-32.

12. Maeda N, Takahashi M, Funahashi T,Kihara S, Nishizawa H, Kishida $\mathrm{K}$, et al. PPAR gamma ligands increase expression and plasma concentrations of adiponectin, an adipose-derived protein. Diabetes 2001;50:2094-9. [CrossRef]

13. Fasshauer M, Kralisch S, Klier M, Lossner U, Bluher M, Klein J, et al. Adiponectin gene expression and secretion is inhibited by interleukin-6 in 3T3-L1 adipocytes. Biochem Biophys Res Commun 2003;301:1045-50. [CrossRef]

14. Teoh H, Quan A, Bang KW, Wang G, Lovren F, Vu V, et al. Adiponectin deficiency promotes endothelial activation and profoundly exacerbates sepsis-related mortality. Am J Pyhsiol Endocrinol Metab 2008;295:658-64. [CrossRef]

15. Ouchi N, Ohishi M, Kihara S, Funahashi T, Nakamura T, Nagaretani H, et al. Association of hypoadiponectinemia with impaired vasoreactivity. Hypertension 2003;42:231-4. [CrossRef]

16. Gearing AJ, Newman W. Circulating adhesion molecules in disease. Immunol Today 1993;14:506-12. [CrossRef]

17. Calandra T, Echtenacher B, Roy DL, Pugin J, Metz CN, Hültner L, et al. Protection from septic shock by neutralization of macrophage migration inhibitory factor. Nat Med 2000;6:164-70. [CrossRef]

18. Kadowaki T, Yamauchi T. Adiponectin and adiponectin receptors. Endoc Rev 2005;26:439-51. [CrossRef] 